\title{
C3-BIOECONOMY
}

Circular and Sustainable Bioeconomy

\section{Estrategia de Bioeconomía y Sostenibilidad en Dcoop a través de la Innovación, Dcoop SCA}

\author{
Belén Barrero Domínguez ${ }^{1}$ y Silvia López Feria ${ }^{1}$
}

Autor de Correspondencia: belen.barrero@dcoop.es / silvia.lopez@dcoop.es

\section{Resumen:}

Europa necesita cambiar radicalmente su manera de producir, consumir, transformar, almacenar, reciclar y eliminar los recursos biológicos para hacer frente al crecimiento de la población mundial, al agotamiento de los recursos, al aumento de la presión sobre el medio ambiente y al cambio climático. La estrategia Europa 2030 propone la bioeconomía como elemento clave para el crecimiento inteligente y ecológico. Los avances en la investigación sobre bioeconomía y la absorción de la innovación permitirán mejorar la gestión de los recursos biológicos renovables y abrir mercados nuevos y diversificados de alimentos y bioproductos.

Siguiendo esta estrategia, Dcoop Sociedad Cooperativa Andaluza, desarrolla su actividad. La sostenibilidad en el sector agroalimentario es uno de los objetivos prioritarios de Dcoop, y para su consecución ha puesto en marcha prácticas sostenibles, tanto en su actividad industrial como productiva, trabajando, además, en varias líneas de innovación agrícola, ganadera, de aprovechamiento de los recursos naturales (subproductos) y de gestión de los residuos producidos durante los procesos productivos que desarrolla. El papel de Dcoop es ser la respuesta que necesita el campo -agricultores y ganaderos- y los consumidores a los desafíos que plantean el mercado global, el cambio climático y los nuevos estilos de vida.

Palabras clave: Bioeconomía, Sostenibilidad, Estrategia, Innovación; Subproductos

\section{Bioeconomy and Sustainability Strategy through Innovation in Dcoop, Dcoop SCA}

\author{
Belén Barrero Domínguez ${ }^{1}$ y Silvia López Feria ${ }^{1}$
}

\section{Abstract:}

Europe needs to radically change it way of producing, consuming, transforming, storing, recycling and disposing of biological resources to cope with global population growth, resource depletion, increasing pressure on the environment and climate change. The Europe 2030 strategy proposes the bioeconomy as a key element for smart and green growth. Advances in bioeconomy research and the uptake of innovation will improve the management of renewable biological resources and open up new and diversified markets for food and bioproducts.

Following this strategy, Dcoop, Andalusian Cooperative Society, is developing its activity. Sustainability in the agrifood sector is one of Dcoop's priority objectives, and to achieve this it has implemented sustainable practices, both in its industrial and production activities. It is also working

1 Dcoop Sociedad Cooperativa Andaluza. Carretera de Córdoba S/N, Antequera, Málaga, 29200 (España), belen.barrero@dcoop.es; silvia.lopez@dcoop.es.

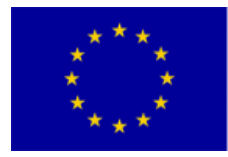

UNIÓN EUROPEA

PROYECTO COFINANCIADO POR ELFONDDO EUROPEO DE DESARROLLO Una manera de hacer Europa

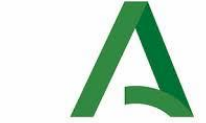

Junta de Andalucía
Andalucía

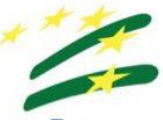

se mueve con Europa 
on various lines of agricultural and livestock research, the use of natural resources and the management of waste produced during its production processes. The role of Dcoop is to be the answer that the farmers, livestock farmers and consumers need to the challenges posed by the global market, climate change and new lifestyles.

Keywords: Bioeconomy, Sustainability, Strategy, Innovation; Subproducts

\section{INTRODUCCIÓN}

Dcoop Sociedad Cooperativa Andaluza, consolidada como uno de los mayores grupos cooperativos alimentarios de segundo grado, desarrolla su principal actividad en los sectores agrícola y ganadero a través de las secciones de Aceite, Aceituna de mesa, Vinos, Ganadería, Cereales, Frutos secos, Suministros, Orujo, Crédito e Industrialización de Leche, constituidas cada una de ellas de manera diferenciadas e independientes, tal y como establece la legislación nacional y autonómica. La misión de Dcoop es trabajar para generar riqueza y empleo en el campo, favoreciendo un intercambio comercial de la producción agraria y ganadera sostenible, innovadora, justa y de calidad en todo el mundo.

Imagen 1. Valores de Dcoop.

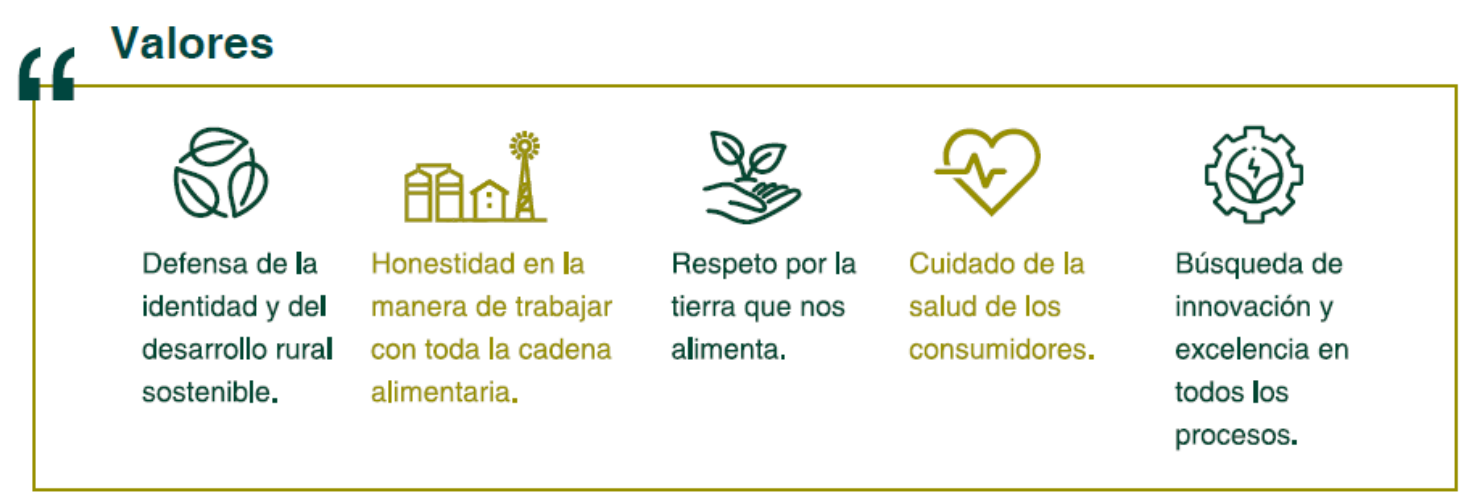

Con el objetivo de dar cumplimiento efectivo a su compromiso con el medio ambiente y los efectos de la contaminación y el cambio climático, desde Dcoop se promueve una cultura de concienciación, respeto y defensa sobre estas materias con la finalidad de tener el mayor alcance posible con la misma. Esta cultura nace a partir de los principios y directrices establecidos en el Acuerdo de París y en el 'Marco de indicadores mundiales para los Objetivos de 
Desarrollo Sostenible y metas de la Agenda 2030 para el Desarrollo Sostenible' de las Naciones Unidas (2020).

Los principios del compromiso medioambiental (Junta de Andalucía, 2018) se concretan en las directrices y objetivos estratégicos establecidos en la Política de Calidad, Seguridad Alimentaria y Medio Ambiente de Dcoop.

Dentro de la cultura medioambiental promovida por Dcoop, se presta especial importancia y atención a todos los aspectos que tienen incidencia, directa e indirecta, con la implementación de procedimientos y procesos que apoyen la economía circular y que mejoren la prevención y gestión de los residuos generados en el desarrollo de los procesos productivos llevados a cabo por el Grupo.

La sostenibilidad en el sector agroalimentario es uno de los objetivos prioritarios de Dcoop, y para su consecución ha puesto en marcha prácticas de sostenibilidad tanto en su actividad industrial como productiva, trabajando, además, en varias líneas de investigación agrícola, ganadera, de aprovechamiento de los recursos naturales y gestión de los residuos producidos durante los procesos productivos que desarrolla.

Las medidas tendentes a la consecución de una economía circular eficiente llevadas a cabo en Dcoop son las siguientes:

- Uso de depuradoras de aguas residuales en la planta de procesamiento de aceitunas de mesa y aderezo para el tratamiento del agua, recuperándose un porcentaje que permite reutilizarla en algunas operaciones de limpieza, y/o devolverlo al medio natural.

- Uso de depuradora en la central láctea de Antequera que realiza un tratamiento al agua resultante de los procesos y permite devolverla a saneamiento público.

- Uso de balsas de evaporación para almacenar el agua residual utilizada en los procesos productivos en la planta de orujo. 
- Uso de una fosa séptica integrada en el sistema de depuración de vertidos para las aguas residuales domésticas del personal que trabaja en la planta de orujo y tratamiento de las mismas a través de un sistema de zanjas filtrantes realizando un proceso de geo-depuración.

- Tratamiento a través de la sección de orujo del alperujo; residuo obtenido durante el proceso productivo para la obtención del aceite; obteniendo aceite de orujo y biomasa, productos que el Grupo comercializa posteriormente. Aprovechando el $100 \%$ de la aceituna y dejando residuo cero en la actividad olivarera.

- Utilización de calderas de biomasa en las almazaras, cuyo combustible principal son los huesos de aceituna previamente obtenidos en los procesos productivos del aceite y la aceituna de mesa y de aderezo.

- Revalorización y utilización de cenizas de la combustión de la biomasa para la elaboración de compost por una empresa autorizada.

- Recuperación de las mermas y arrastres generados durante la producción del vino y comercialización en el mercado como vino de destilación.

- Compactación de residuos en línea.

- El resto de residuos generados, como son: cartón, plástico, latas, vidrio, basura orgánica, hojarasca, lodos, cenizas y cartón peligroso (por contener productos químicos), se encuentran regulados y controlados por los gestores de residuos autorizados.

- Creación de líneas de tratamiento o reutilización de los productos obsoletos.

- Aplicación de nuevas técnicas de packaging: reducción de gramajes, uso de materiales reciclados en el proceso y reciclabilidad total del packaging.

Asimismo, Dcoop ha adoptado una serie de medidas para la reducción del consumo energético en sus instalaciones:

- Sustitución de maquinaria con menor consumo y aumento de eficiencia. 
- Sustitución de cubiertas en naves colocando paneles sándwich mejorando el aislamiento térmico de los edificios.

- Instalación de translúcidos en cubiertas.

- Aumento de la eficiencia de las líneas de fabricación.

- Autoconsumo de la cáscara de almendra en diferentes industrias.

\section{EJEMPLOS DE APLICACIÓN DE LA BIOECONOMÍA: PROYECTOS DE I+D+i DE DCOOP}

Desde hace más de una década, tras la creación del Departamento de l+D+i de Dcoop, se definieron, en colaboración con todas las secciones del Grupo, una política de trabajo para el desarrollo de una producción agraria y ganadera sostenible, innovadora, justa y de calidad que a su vez diera respuesta a las necesidades del campo-agricultores y ganaderos- y de los consumidores frente a los desafíos que plantean el mercado global, el cambio climático y los nuevos estilos de vida.

Como resultado, se obtuvo el Plan de Innovación de Dcoop que se basa en cuatro pilares estratégicos en los que se enmarcan los proyectos de I+D+i del Grupo:

1) Calidad, trazabilidad y seguridad alimentaria.

2) Economía circular, sostenibilidad y aprovechamiento de subproductos.

3) Industria 4.0 (modernización de los procesos productivos del campo a la mesa).

4) Desarrollo de nuevos productos saludables orientados a las nuevas necesidades de nuestra sociedad.

A continuación, se plasman varios proyectos de l+D+i en los que participa Dcoop y que están centrados en la aplicación de la Bioeconomía: 


\subsection{BIOECOLIVA: Mejora de la eficiencia y sostenibilidad de las almazaras bajo el paradigma de la bioeconomía}

BIOECOLIVA: Mejora de la eficiencia y sostenibilidad de las almazaras bajo el paradigma de la bioeconomía (Grupo Operativo Autonómico) surgió con el objetivo de mejorar la toma de decisiones en las almazaras, de forma que se contribuyese económica, social y medioambientalmente al entorno rural en el que se asientan, resultando necesario identificar factores que permitiesen establecer sinergias y acciones de mejora adaptadas a los diferentes tipos de almazaras para que mejoren su eficiencia y sostenibilidad. Para ello, se propuso el diseño de una metodología compleja (Análisis clúster, Análisis Envolvente de Datos -DEA- con distancias econométricas, regresión múltiple, análisis multicriterio -AHP-) y novedosa que permitiese alcanzar la eficiencia técnica y el nivel de calidad de la producción e incorporación de prácticas respetuosas con el entorno, que contribuyesen a la cohesión social.

BIOECOLIVA ha permitido llevar a cabo un exhaustivo análisis sobre la eficiencia y sostenibilidad del sector cooperativo olivarero e identificar aspectos clave de mejora, tanto desde el punto de la eficiencia técnica como desde el punto de vista de la sostenibilidad ambiental. Los resultados podrían ser aplicados como guía para la mejora de las cooperativas. En el proyecto se han detectado los puntos fuertes del sector, pero también importantes acciones de mejora, cuya aplicación requerirá de la participación de todas las cooperativas analizadas.

\subsection{DOSAOLIVAR: Dosificación de fitosanitarios en olivar}

El objetivo del proyecto DOSAOLIVAR: Dosificación de fitosanitarios en olivar (Grupo Operativo Autonómico) ha sido el desarrollo de un sistema que permita la planificación y aplicación óptimas de los tratamientos fitosanitarios acorde a la situación y características del cultivo. Con esta planificación se potenciará la eficiencia técnica, ambiental, energética y económica, y se mitigarán las emisiones que afectan al cambio climático en el sector oleícola. 
La herramienta desarrollada en DOSAOLIVAR está generada con una base científica y tecnológica, además es dinámica, sencilla y actualizable, lo que facilita su uso a los olivicultores para la toma de mejores decisiones en cuanto los tratamientos fitosanitarios. Con el empleo de este sistema se podrá conseguir una aplicación de estos tratamientos en el olivar más sostenible.

Los Grupos Operativos Autonómicos BIOECOLIVA y DOSAOLIVAR han sido financiados por la Consejería de Agricultura, Pesca, Ganadería y Desarrollo Sostenible y la Unión Europea mediante fondos FEADER.

\section{Imagen 2. Logos de los organismos financiadores de los proyectos BIOECOLIVA y}

DOSAOLIVAR.

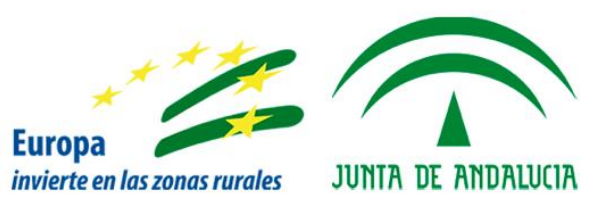

$\begin{array}{cl}\star^{\star \star \star \star} & \text { UNIÓN EUROPEA } \\ \star_{\star \star \star}^{\star \star} & \begin{array}{l}\text { Fondo Europeo Agricola } \\ \text { de Desarrollo Rural }\end{array}\end{array}$

\subsection{ACTILIFE: Alimentos para una población saludable y deportista}

La cuarta línea estratégica del Departamento de I+D+i de Dcoop se centra en el desarrollo de nuevos productos innovadores dirigidos a proporcionar beneficios para la salud y con propiedades de alto valor añadido para el consumidor. Esta línea va de la mano del aprovechamiento y revalorización de los subproductos de las distintas cadenas de producción que posee Dcoop y, por tanto, de la aplicación de los principios de economía circular y sostenibilidad.

Podemos identificar varios proyectos de innovación donde se desarrollan nuevos productos/ingredientes para consumo humano basados en la revalorización y aprovechamiento de los subproductos de los procesos productivos del aceite de oliva virgen, queso de cabra y/o almendra. Dentro 
de estos estudios, se identifica una población objetivo y se trabaja para mejorar el estado de salud de ese grupo o su estilo de vida en cuanto a la alimentación.

En el proyecto ACTILIFE: Alimentos para una población saludable y deportista (CDTI-CIEN), la población objeto de estudio son los deportistas, donde se busca complementar su alimentación en base a la actividad física que realicen y mejorar así algún aspecto fisiológico (rendimiento, tiempo de recuperación, restablecimiento de minerales, hidratación...). Los subproductos evaluados en ACTILIFE proceden de diversas fuentes agroindustriales: hueso y semilla de aceituna, suero de queso de cabra, permeado de leche de cabra y diferentes variedades de almendra.

Una de las fuentes de mayor interés de aplicación en la nutrición deportiva está siendo el suero obtenido del proceso productivo del queso de cabra. Se está trabajando en su transformación para incorporarlo en un formato atractivo y organolépticamente deseable para el consumidor. También se está estudiando sobre un ingrediente antioxidante procedente de la producción de aceituna de mesa para añadir a otras matrices alimentarias y aumentar la capacidad antioxidante de las mismas, tan relevante en los deportistas. En paralelo, se está desarrollando una herramienta de gestión digital que aportará información nutricional para el cumplimiento de todos los requerimientos nutricionales y de salud de interés en la práctica deportiva.

Imagen 3. Suero de queso de cabra seco en estufa a vacío.

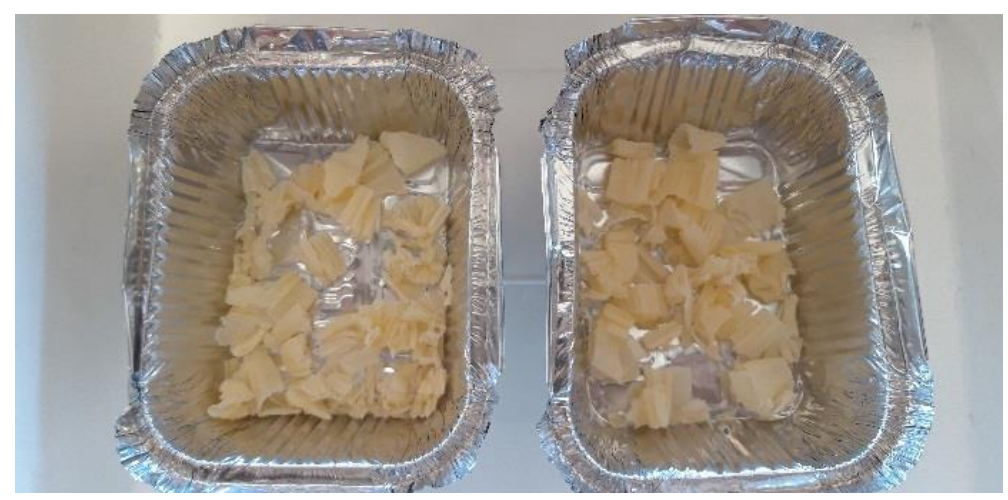


Imagen 4. Suero molido tras secado en estufa a vacío.

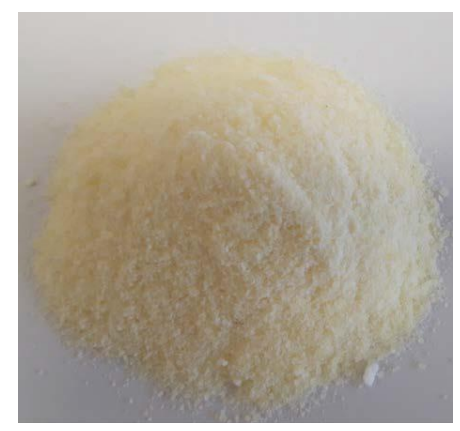

\subsection{TECFOOD: Nuevos alimentos tecnológicos saludables para las nuevas necesidades sociales}

En la misma línea se encuentra TECFOOD: Nuevos alimentos tecnológicos saludables para las nuevas necesidades sociales (CDTI-CIEN), cuyo objetivo es lograr aumentar la capacidad innovadora de la industria agroalimentaria a través del desarrollo de nuevos procesos y productos alimentarios de mayor valor añadido, así como potenciar el desarrollo de sectores emergentes y de alto contenido tecnológico como la impresora 3D de alimentos. Todo ello con el fin de satisfacer las necesidades y expectativas de unos consumidores finales y de una sociedad que demanda unos productos alimentarios con un perfil nutricional saludable y con nuevas texturas, sin olvidar las nuevas necesidades de la sociedad cada vez más envejecida.

En este proyecto Dcoop trabaja con materias primas y subproductos del olivar. Tras caracterizar todas sus materias se ha generado un ingrediente (coproducto de la producción de aceite de oliva virgen) de aplicación a otras matrices alimentarias para aportar valor nutricional y tecnológico. Por otro lado, Dcoop se encuentra desarrollando un producto innovador derivado del aceite de oliva virgen que, junto con los subproductos derivados de la aceituna de mesa, están en estudio y ensayo para poder ser utilizados en la nueva tecnología de impresión 3D de alimentos. 
Imagen 5. Ejemplo Impresión 3D con Untable de aceite de oliva virgen.

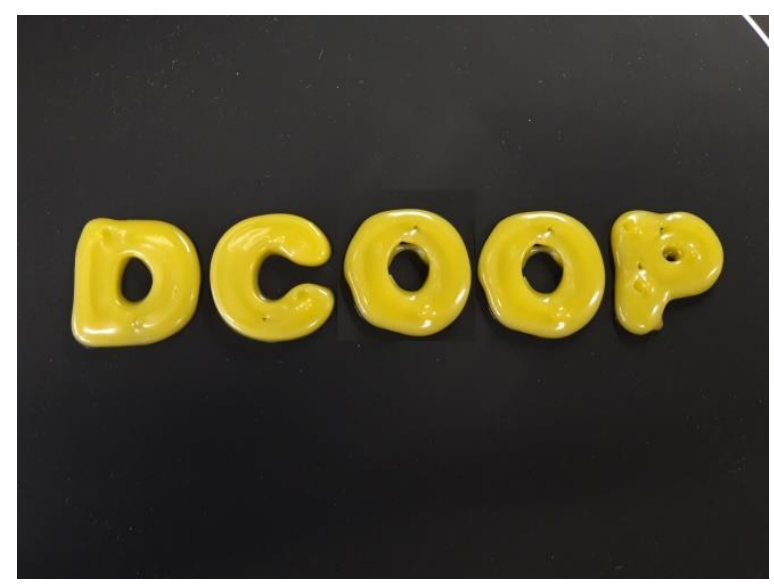

La cooperativa de segundo grado será pionera en llevar sus alimentos e ingredientes a esta tecnología que, en un futuro será capaz de diseñar platos saludables para mantener una dieta equilibrada y cocinarlos según las necesidades específicas de cada consumidor.

\subsection{TERÁTROFO: Generación de nuevos ingredientes y alimentos beneficiosos} dirigidos a condiciones de riesgo y al bienestar global de personas con cáncer colorrectal

El objetivo principal del proyecto TERÁTROFO: Generación de nuevos ingredientes y alimentos beneficiosos dirigidos a condiciones de riesgo y al bienestar global de personas con cáncer colorrectal (CDTI-CIEN) es la investigación y desarrollo experimental de nuevos productos de consumo alimentario e ingredientes más seguros y beneficiosos para prevenir el riesgo de cáncer colorrectal (CCR) y coadyuvar positivamente en la respuesta nutricional al tratamiento del mismo.

Teniendo en cuenta la bibliografía existente, así como el know-how creado en Dcoop para el desarrollo de otros proyectos de investigación, se han seleccionado aquellas materias con las características más adecuadas para el desarrollo del TERÁTROFO. A parte de la selección de materias primas, se ha comenzado a estudiar nuevos métodos destinados a la obtención de subproductos de la producción de aceite de oliva y aceituna de mesa con propiedades sustancialmente mejoradas y funcionalidades específicas que 
permitan elaborar productos que sean empleados por las personas con riesgo de desarrollar CCR O las personas que están sometiéndose a tratamiento oncológico.

\subsection{FOOD4STROKE: Alimentos con efecto neuroprotector para un envejecimiento activo de la sociedad}

FOOD4STROKE: Alimentos con efecto neuroprotector para un envejecimiento activo de la sociedad (CDTI-CIEN) nace con el objetivo de obtener ingredientes con efecto neuroprotector a partir de materias primas procedentes del sector agroalimentario para el desarrollo de alimentos y complementos alimenticios que permitan mejorar la calidad de vida y la salud de los consumidores, previniendo enfermedades cerebrovasculares de gran incidencia en la sociedad, como el ictus, a través de una nutrición personalizada.

En este proyecto Dcoop está llevando a cabo diversos análisis para la caracterización de sus materias primas y también está realizando ensayos en olivar. Todo ello con el fin de formular posibles ingredientes con efecto neuroprotector a partir de subproductos del olivar y de la industria láctea de cabra, existiendo además la posibilidad de combinación con otras materias primas aportadas por otros miembros del consorcio.

En estos cuatro proyectos CDTI-CIEN (ACTILIFE, TECFOOD, TERÁTROFO y FOOD4STROKE) es esencial la sinergia y colaboración entre las empresas alimentarias. Por ello, Dcoop trabaja con todas las materias propuestas para que cualquier ingrediente de interés para los demás participantes establezca la posibilidad de colaboración y de desarrollo de un nuevo producto adaptado a las necesidades de la población.

Imagen 6. Logos de los organismos financiadores de los proyectos ACTILIFE, TECFOOD,

TERÁTROFO Y FOOD4STROKE.

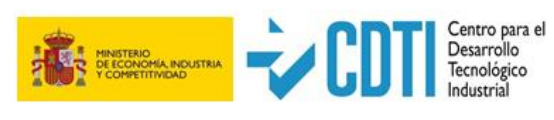




\subsection{OLIVALPORC: Soluciones tecnológicas y aprovechamiento sostenible para una alimentación porcina saludable, funcional y segura que mejore las propiedades de la carne}

Además del enfoque de desarrollo de nuevos productos directos para consumo humano, en Dcoop se trabaja también desde la base de la cadena alimentaria incidiendo, a través de varios proyectos, en la alimentación animal. Concretamente en el sector porcino se ha enmarcado el proyecto OLIVALPORC: Soluciones tecnológicas y aprovechamiento sostenible para una alimentación porcina saludable, funcional y segura que mejore las propiedades de la carne (CDTI en Cooperación). El objetivo de este proyecto ha sido el desarrollo de nuevas formulaciones de piensos para las distintas fases de la producción porcina (cerdos blancos en las fases de crecimiento-cebo y cerdas ibéricas gestantes), permitiendo una economía circular y ligando la olivicultura y la ganadería en las regiones mediterráneas a través de la incorporación del orujo graso seco (OGS). Durante el transcurso del proyecto se ha comprobado que la inclusión de este coproducto del olivar como materia prima proporciona propiedades mejoradas en la alimentación porcina e incide positivamente en la salud de los animales y en la producción de carne.

Imagen 7. Cerdas ibéricas gestantes alimentadas con el pienso diseñado para los ensayos.

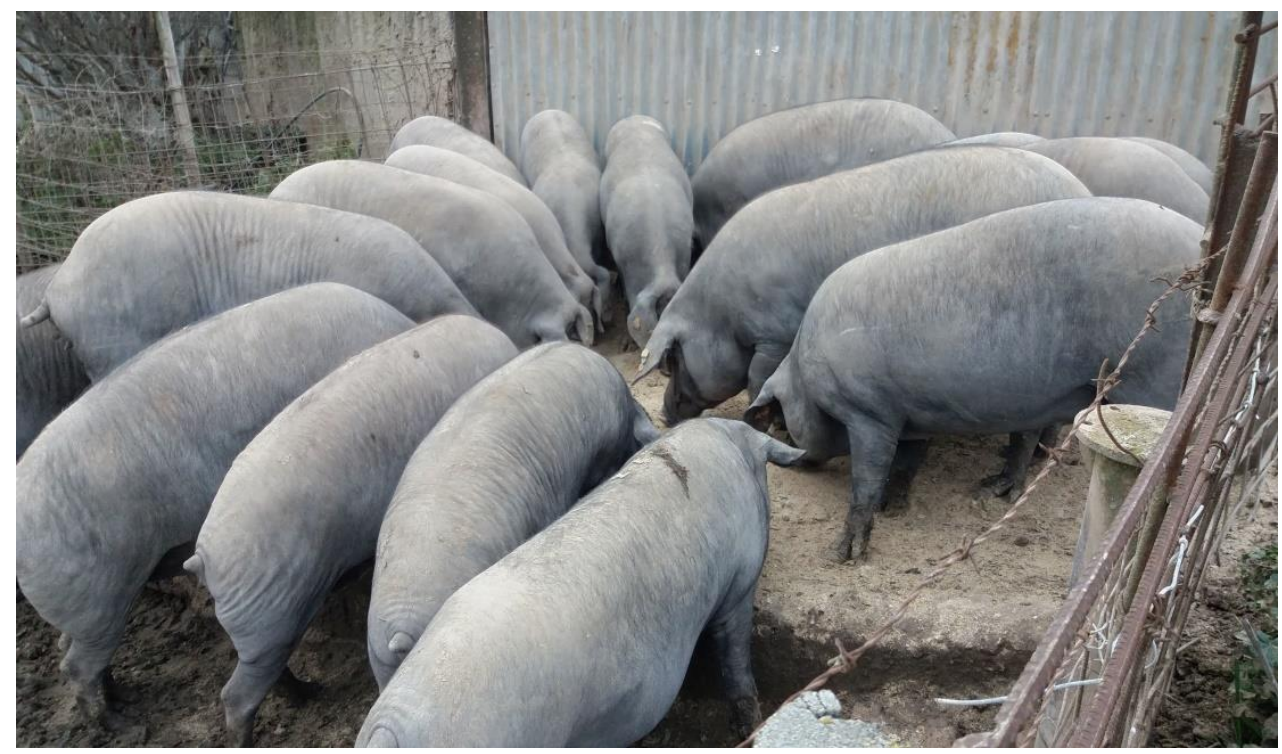


Este proyecto ha contribuido en la mejora de la posición competitiva de Dcoop, ya que la cooperativa ha cubierto su objetivo comercial y estratégico al valorizar un subproducto generado durante el proceso de producción del aceite de oliva como materia prima para la alimentación porcina. De esta forma, Dcoop puede proporcionar información técnica sobre su correcta aplicación e introducción en nutrición porcina, abriendo así una nueva línea de comercialización complementaria.

\subsection{DEALMALTEA: Valoración de la capota de almendra para alimentación animal}

Por último, y también englobado en la línea estratégica de economía circular, sostenibilidad y aprovechamiento de subproductos se encuentra el proyecto DEALMALTEA: Valoración de la capota de almendra para alimentación animal (CDTI en Cooperación), cuyo objetivo principal es la valorización de la capota de almendra a través de su inclusión en la alimentación de pequeños rumiantes (caprino de leche y ovino de carne). En el trascurso del proyecto se han determinado las propiedades nutricionales de la capota de distintas variedades de almendra, definido el momento óptimo de obtención del subproducto en consonancia con la calidad óptima de la almendra, evaluado la forma de aplicación de la capota de almendra en la alimentación animal (triturada, en harina, pelletizada, etc.) y el porcentaje de inclusión dentro de la formulación de los piensos. Actualmente, se están analizando los efectos de los piensos sobre los animales y sobre sus productos: efecto sobre la leche en cabras y sobre la carne en corderos. 
Imagen 8. Almendras con su capota.

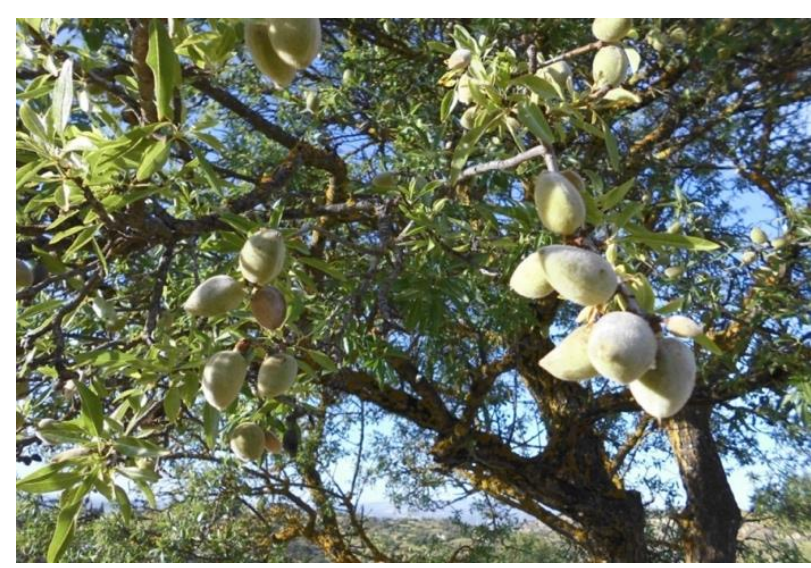

Los proyectos CDTI OLIVALPORC y DEALMALTEA están cofinanciados por el Fondo Europeo de Desarrollo Regional (FEDER), a través del Programa Operativo Plurirregional de España 2014-2020.

Imagen 9. Logos de los organismos financiadores de los proyectos OLIVALPORC y DEALMALTEA.

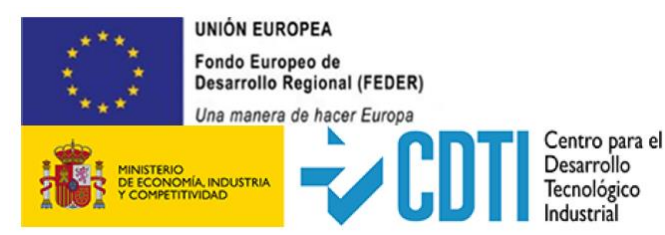

\section{REFERENCIAS}

JUNTA DE ANDALUCÍA (2018). Estrategia Andaluza de Bioeconomía Circular. https://www.juntadeandalucia.es/export/drupaljda/Estrategia Andaluz a_Bioeconomia_Circular EABC 18.09.2018.pdf

NACIONES UNIDAS (2020). Marco de indicadores mundiales para los Objetivos de Desarrollo Sostenible y metas de la Agenda 2030 para el Desarrollo Sostenible.

https://unstats.un.org/sdgs/indicators/Global\%20Indicator\%20Framework \%20after\%202020\%20review Spa.pdf 